\title{
Exploring the characteristics of processed meat consumers and facilitators of reducing processed meat consumption
}

\author{
L. Sanderson and J.K. Lodge \\ Department of Applied Sciences, Northumbria University, Newcastle Upon Tyne, NE1 8ST, UK
}

There is an increasing body of evidence regarding the adverse health effects of processed meat ${ }^{(1,2)}$ and therefore a reduction in its consumption is of increasing public health interest. Current government guidelines, which recommend no more than $70 \mathrm{~g}$ a day of processed or red meat ${ }^{(3)}$, remain unmet (current average consumption: $80 \mathrm{~g} /$ day for women, and $97 \mathrm{~g} / \mathrm{day}$ for men) and the existing research on attitudes towards processed meat, and consumer's knowledge and behaviours towards eating less processed meat, is limited. The aim of this pilot study was, to explore a range of characteristics and dietary intake of processed meat consumers and consider potential facilitators for influencing a reduction in consumption, using a semi- qualitative approach.

Male and female adults $(n=20)$ aged 25-55 years completed a four day food diary and semi- qualitative questionnaire which assessed characteristics, such as: participants nutritional knowledge; their attitudes towards processed meat and limiting its consumption, through open- ended questions.

Consumers who reported a high consumption of processed meat ( $>3$ portions/week) had a higher intake of all macro nutrients than those who reported a low consumption of processed meat ( $\leqslant 3$ portions/week), although not statistically significant. High consumers did however have a significantly higher percentage RNI intake of zinc $(\mathrm{P}=0.02)$. High consumers also achieved a higher score when tested on their awareness of the associated health risks of processed meat intake, though not significant.

The key themes present highlighted that convenience, cost, availability, perceived nutritional value of processed meat products as well as appealing sensory qualities such as taste and texture were factors influencing the consumption of processed meat. All respondents acknowledged the need to reduce their processed meat consumption however a problem identified was the lack of accessible alternative options to processed meat. The table illustrates the suggested methods to facilitate a reduction of processed meat consumption:

\begin{tabular}{l}
\hline Participant suggestions to reduce processed meat consumption \\
Improve education to increase awareness of adverse health effects \\
Increase price \\
Reduce availability of processed meat products \\
Improve accessibility to healthier alternatives i.e. improve promotion, reduce price \\
Increase awareness of animal welfare issues
\end{tabular}

The data collected displayed the perceived facilitating factors and current barriers to reducing processed meat consumption and associated characteristics of processed meat consumers. This justifies the suggestion for further research into consumer attitudes towards eating less processed meat, which could provide cultural information for the future development of public health initiatives and interventions to reduce processed meat consumption in the UK.

1. Wiseman M. (2008) Proc $N$ Soc 67, 253-256.

2. Micha R., Michas G., Lajous M. \& Mozaffarian D. (2013) BMC Med 11, 136

3. SACN (2010) Iron and Health. Available at: https://www.gov.uk/government/uploads/system/uploads/attachment_data/file/339309/ SACN_Iron_and_Health_Report.pdf 